\section{(D) Check for updates}

Cite this: Analyst, 2017, 142, 1756

\title{
Surfactant exfoliated 2D hexagonal Boron Nitride (2D-hBN) explored as a potential electrochemical sensor for dopamine: surfactants significantly influence sensor capabilities $\uparrow$
}

\author{
Aamar F. Khan, (D) a Dale A. C. Brownson, (D) ${ }^{a}$ Christopher W. Foster, (D) ${ }^{a}$ \\ Graham C. Smith (D) ${ }^{b}$ and Craig E. Banks (D) $*^{a}$
}

Surfactant exfoliated 2D hexagonal Boron Nitride (2D-hBN) nanosheets are explored as a potential electrochemical sensing platform and evaluated towards the electroanalytical sensing of dopamine (DA) in the presence of the common interferents, ascorbic acid (AA) and uric acid (UA). Surfactant exfoliated 2D-hBN nanosheets (2-4 layers) fabricated using sodium cholate in aqueous media are electrically wired via a drop-casting modification process onto disposable screen-printed graphite electrodes (SPEs). We critically evaluate the performance of these 2D-hBN modified SPEs and demonstrate the effect of 'mass coverage' towards the detection of DA, AA and UA. Previous studies utilising surfactant-free (pristine) 2D-hBN modified SPEs have shown a beneficial effect towards the detection of DA, AA and UA when compared to the underlying/unmodified graphite-based electrode. We show that the fabrication route utilised to prepare $2 \mathrm{D}-\mathrm{hBN}$ is a vital experimental consideration, such that the beneficial effect previously reported is considerably reduced when surfactant exfoliated 2D-hBN is utilised. We demonstrate for the first time, through implementation of control experiments in the form of surfactant modified graphite electrodes, that sodium cholate is a major contributing factor to the aforementioned detrimental behaviour. The significance here is not in the material per se, but the fundamental knowledge of the surfactant and surface coverage changing the electrochemical properties of the material under investigation. Given the wide variety of ionic and non-ionic surfactants that are utilised in the manufacture of novel 2D materials, the control experiments reported herein need to be performed in order to de-convolute the electrochemical response and effectively evaluate the 'underlying surface/surfactant/2D materials' electrocatalytic contribution.

Received 23rd February 2017, Accepted 10th April 2017

DOI: $10.1039 / c 7 a n 00323 d$

rsc.li/analyst

\section{Introduction}

Dopamine (DA), a catecholamine that exists in the extracellular fluid of the central nervous system, is crucial to the routine functioning of numerous biological systems. ${ }^{1-3}$ Irregular levels of this compound can lead to neurological disorders such as Schizophrenia, attention deficit hyperactivity disorder (ADHD) and Parkinson's disease. ${ }^{1,4,5}$ Although a range of sensing platforms are continuously being developed for DA, electrochemical derived sensors attract significant attention due to

\footnotetext{
${ }^{a}$ Faculty of Science and Engineering, Manchester Metropolitan University, Chester Street, Manchester M1 5GD, UK. E-mail: c.banks@mmu.ac.uk; http://www.craigbanksresearch.com; Fax: +(0)1612476831; Tel: +(0)1612471196

${ }^{b}$ Faculty of Science and Engineering, Department of Natural Sciences, University of Chester, Thornton Science Park, Pool Lane, Ince, Chester CH2 4NU, UK

$\dagger$ Electronic supplementary information (ESI) available. See DOI: 10.1039/ c7an00323d
}

their ability to convert chemical information into an electrical signal and through careful design can give rise to sensitive, selective, experimentally simple, portable and low cost sensor devices. ${ }^{6,7}$ Resultantly, numerous efforts towards the electrochemical detection of DA are undertaken, ${ }^{8-10}$ however DA's coexistence with ascorbic acid (AA) often results in overlapping voltammetric signals and complicated voltammetry, hindering detection. $^{11}$ Research is currently focused on imparting improved analytical responses for these two compounds. ${ }^{12,13}$ Specifically, the utilisation of metallic (gold, platinum) or carbon based (glassy carbon, GC) electrodes are often prone to surface fouling and do not adequately allow for the differentiation between the oxidation peak potentials of DA and AA, and in certain sample matrixes, uric acid (UA) is also problematic. ${ }^{14,15}$ As such, an electrode material that improves the electrochemical detection capabilities of DA, whilst efficiently differentiating the peaks of common interferents and that is not prone to surface fouling, is highly sought. ${ }^{16}$ 
Previous studies have utilised 2D materials towards the detection of DA, AA and UA. ${ }^{3,17-19}$ Han et al. ${ }^{17}$ observed that a GC electrode modified with chitosan-graphene allowed for the simultaneous detection of DA, AA and UA. They reported three distinct oxidation peaks at ca. $0.12,0.24$ and $0.33 \mathrm{~V}$, which were attributed to AA, DA and UA respectively. ${ }^{17}$ Conversely, an unmodified GC electrode exhibited a convoluted single peak at ca. $0.49 \mathrm{~V}$. In other research, Sheng et al. ${ }^{18}$ found the simultaneous detection of DA, AA and UA was viable when utilising nitrogen-doped graphene modified upon a GC electrode, whilst Tian et al. ${ }^{19}$ explored the simultaneous detection of AA, DA and UA utilising a GC electrode after modification with graphene (decorated with gold nanoparticles) and found the simultaneous detection of these compounds was also viable (at oxidation potentials of $c a$. $0.22,0.43$ and $0.55 \mathrm{~V}$ respectively). Such a response is significantly beneficial over that of a bare/unmodified GC electrode, where a single oxidation peak was observed at ca. $0.35 \mathrm{~V}$. Moreover, Sun et al. ${ }^{3}$ utilised gold nanoparticle decorated $\mathrm{MoS}_{2}$ nanosheet modified GC electrodes and reported the peak separation between DA/AA and DA/UA at $c a$. 0.15 and $0.14 \mathrm{~V}$ respectively; thus allowing for the simultaneous detection of these compounds, which is not possible at the unmodified GC electrode. A summary of the different 2D materials utilised in the detection of DA can be found in Table 1. What is evident from examination of previous literature, is that researchers often neglect two serious considerations that are vital to understanding the true performance of a given nanomaterial towards the resulting sensor: (i) the implications that the fabrication route may impart onto the observed voltammetry; (ii) the effects of the novel material's 'mass coverage' on their chosen supporting material. Both of these factors are critical oversights and can often lead to the properties of new and novel 2D materials being wrongly reported.

We have previously reported that surfactant-free (pristine) 2D hexagonal Boron Nitride (2D-hBN) nanosheets exhibit an electrocatalytic behaviour towards the sensing of DA when immobilised upon screen-printed graphite electrodes (SPEs). ${ }^{20}$ Pristine 2D-hBN reduced the potential required for the electrochemical oxidation of DA by $c a .90 \mathrm{mV}$ and increased the peak current/signal in comparison to unmodified/underlying SPEs. ${ }^{20}$ Although the simultaneous detection of DA and AA was not viable, the simultaneous detection of DA and UA was achieved with this novel approach utilising $2 \mathrm{D}-\mathrm{hBN}$ as the sensing platform. ${ }^{20}$ When utilising the 2D-hBN modified SPEs, a beneficial increase in the peak separation between DA and UA was reported ( $c a .50 \mathrm{mV}$ increase $v s$. unmodified) whilst the 2D-hBN based sensor offered improved peak currents/signal in contrast to an unmodified SPE. ${ }^{20}$ Interestingly, no such beneficial response was observed for 2D-hBN when it was modified/ supported on GC and boron doped diamond (BDD) underlying electrodes. The observed beneficial response at 2D-hBN modified SPEs was attributed to the interaction between 2D-hBN and the roughness of the underlying SPE, whereas the comparatively smooth surface of a GC electrode hindered this. ${ }^{20}$ This initial work demonstrated the importance of considering the influence of additional factors (other than that of the 2D material chosen) and their contribution to the observed electrochemical response. There are now many routes to fabricate $2 \mathrm{D}$ materials, and surfactant exfoliation is a common and favourable approach due to its simplicity. ${ }^{21}$

To the best of our knowledge, surfactant exfoliated 2D-hBN has yet to be explored towards the detection of DA in the presence of AA and UA. As such, we now seek to demonstrate that care needs to be taken, not only with the choice of $2 \mathrm{D}$ material, but also when considering its fabrication route, which can dramatically affect the electrochemical performance.

Herein, we consider for the first time, the electrochemical oxidation of DA, AA and UA utilising surfactant (sodium cholate) exfoliated 2D-hBN nanosheet modified SPEs. Furthermore, control experiments, in the form of surfactant

Table 1 A comparison of various 2D materials utilised towards the detection of DA

\begin{tabular}{|c|c|c|c|c|c|}
\hline Electrode material & $\begin{array}{l}\text { Electrocatalytic } \\
\text { (with regards to) }\end{array}$ & Dopamine LOD $(\mu \mathrm{M})$ & $\begin{array}{l}\text { Explored in the } \\
\text { presence of: }\end{array}$ & $\begin{array}{l}\text { Electrochemical } \\
\text { method }\end{array}$ & Ref. \\
\hline AuNPs@MoS & GC & 0.05 & $\mathrm{UA}, \mathrm{AA}$ & DPV & 3 \\
\hline Chitosan-graphene & GC & 1.00 & UA, AA & DPV & 17 \\
\hline AuNPs- $\beta$-CD-graphene & $\mathrm{GC}$ & 0.15 & $\mathrm{UA}, \mathrm{AA}$ & SWV & 19 \\
\hline Functionalised graphene & Bare graphite & 0.25 & $\mathrm{UA}, \mathrm{AA}$ & DPV & 36 \\
\hline PtNPs@MoS 2 & GC & 0.17 & UA & DPV & 37 \\
\hline Nitrogen doped graphene & GC & 0.25 & $\mathrm{UA}, \mathrm{AA}$ & DPV & 18 \\
\hline AuNPs@MoS ${ }_{2}$ & GC & 0.08 & $\mathrm{AA}$ & DPV & 38 \\
\hline Graphene & GC & 2.64 & $\mathrm{AA}$ & DPV & 39 \\
\hline GNS-CNTs/ $\mathrm{MoS}_{2}$ & GC & 0.05 & - & DPV & 40 \\
\hline $\mathrm{MoS}_{2}$-RGO & GC & 0.94 & $\mathrm{AA}$ & DPV & 41 \\
\hline Pristine 2D-hBN & SPE & 0.65 & $\mathrm{UA}, \mathrm{AA}^{a}$ & DPV & 20 \\
\hline Surfactant exfoliated 2D-hBN & SPE & 1.57 & $\mathrm{UA}, \mathrm{AA}^{a}$ & DPV & This work \\
\hline
\end{tabular}

Key: PtNP@MoS 2 , platinum nanoparticle modified molybdenum disulfide; AuNP@MoS 2 , gold nano-particle modified molybdenum disulfide; GNS-CNTs/ $\mathrm{MoS}_{2}$, molybdenum disulfide flowers placed on graphene nanosheets and multiwalled carbon nanotubes; MoS - -RGO, molybdenum disulphide - reduced graphene oxide; gold nanoparticle modified molybdenum disulfide; AuNPs- $\beta$-CD-graphene, gold nanoparticles- $\beta$-cyclodextrin-graphene; 2D-hBN, 2D hexagonal boron nitride; GC, glassy carbon electrode; CPE, carbon paste electrode; SPE, screen-printed graphite electrode; Functionalised graphene, synthesised by solvothermal reduction of colloidal dispersions of graphite oxide; UA, uric acid; AA, ascorbic acid; DPV, differential pulse voltammetry; SWV, square wave voltammetry. ${ }^{a}$ DA and AA signal de-convolution is not possible upon simultaneous detection. 
modified (in the absence of 2D-hBN) graphitic electrodes and mass-coverage studies are implemented in order to de-convolute the true electrochemical response, such that we aim to uncover fundamental knowledge concerning the influence of both the surfactant and surface coverage on the resulting electrochemical properties of the material under investigation. The effect of surfactant-exfoliated 2D-hBN, to the best of our knowledge, has not yet been explored in this manner.

\section{Experimental section}

All chemicals were of analytical grade and were used as received from Sigma-Aldrich without any further purification. All solutions were prepared with deionised water of resistivity not less than $18.2 \mathrm{M} \Omega \mathrm{cm}$ and were vigorously degassed prior to electrochemical measurements with high purity, oxygen free nitrogen.

Voltammetric measurements were performed using an 'Autolab PGSTAT 101' (Metrohm Autolab, The Netherlands) potentiostat. All measurements were conducted using a conventional three electrode system. Screen-printed graphite electrodes (SPEs) with a $3.1 \mathrm{~mm}$ diameter were used as working electrodes. ${ }^{22,23}$ Surfactant exfoliated 2D-hBN modified working electrodes were prepared by drop-casting aliquots of a sodium cholate hydrate (sodium cholate) solution containing 2D-hBN onto the required working electrode with a micropipette. After 30 minutes, the water, containing sodium cholate, had completely evaporated (at ambient temperature) and the modified electrodes were ready for use (or for further modification). A platinum wire and a saturated calomel electrode (SCE) were used as counter and reference electrodes respectively.

The SPEs consist of a graphite working electrode and were fabricated in-house. Fabrication details and appropriate characterisation can be found in the ESI $\dagger$ to allow future researchers to benchmark the system utilised.

The surfactant exfoliated (surfactant encapsulated) 2D-hBN utilised herein was fabricated in-house. Utilising a method reported previously by Kurapati et al. $;^{24}$ bulk Boron Nitride was procured from Sigma Aldrich (used as received), after which it was sonicated in an aqueous solution (water, $\mathrm{pH}$ 7.6) containing sodium cholate, to induce liquid phase exfoliation. Interested readers are directed to the ESI $\dagger$ for further details on the surfactant based liquid exfoliation, sonication and centrifugation methodology utilised. The resultant product comprised surfactant (sodium cholate) exfoliated 2D-hBN nanosheets sodium cholate $\left(2 \mathrm{~g} \mathrm{~L}^{-1}\right)$ in an aqueous solution. Note that where surfactant control experiments were utilised, a $2 \mathrm{~g} \mathrm{~L}^{-1}$ solution of sodium cholate was prepared and utilised in the absence of 2D-hBN (i.e. 2D material not present). A schematic representation of the surfactant encapsulated $2 \mathrm{D}-\mathrm{hBN}$ is represented in ESI Scheme $1, \dagger$ where it is observed that the hydroxyl groups of sodium cholate may be electroactive and we explore this further throughout the manuscript. ${ }^{25}$

Physicochemical characterisation of the surfactant exfoliated 2D-hBN was carried out utilising transmission electron microscopy (TEM), scanning electron microscopy (SEM), ultra violet (UV)-visible Spectroscopy, energy-dispersive X-ray (EDX) analysis, and X-ray photoelectron spectroscopy (XPS). Details of the equipment (and operative conditions) utilised are reported in the ESI. $\dagger$

\section{Optical band gap calculation (later applied towards 2D-hBN)}

The Tauc equation: $\omega^{2} \varepsilon=\left(h \omega-E_{\mathrm{g}}\right)^{2}$ was utilised to determine the optical band gap $E_{\mathrm{g}}$ for surfactant exfoliated $2 \mathrm{D}-\mathrm{hBN}{ }^{26}$ Where $\varepsilon$ is the optical absorbance, $h$ is Planck's constant, and $\omega=2 \pi / \lambda$ is the angular frequency of the incident radiation $(\lambda$ is the wavelength). Thus, a plot of $\varepsilon^{1 / 2} / \lambda$ versus $1 / \lambda$ results in a straight line at the absorption range. Hence, the intersection point with the $x$ axis is $1 / \lambda_{\mathrm{g}}$ ( $\lambda_{\mathrm{g}}$ is defined as the gap wavelength). Finally, the optical band gap can be calculated utilising the equation $E_{\mathrm{g}}=h c / \lambda_{\mathrm{g}}$.

\section{Physicochemical characterisation}

We have previously utilised TEM, SEM, XRD, XPS, EDX UVvisible spectroscopy and Raman spectroscopy to comprehensively characterise the surfactant exfoliated $2 \mathrm{D}-\mathrm{hBN} .^{27}$ Interested readers are referred to the $\mathrm{ESI} \dagger$ for further information. A TEM image is depicted in Fig. 1A and indicates that the surfactant exfoliated $2 \mathrm{D}-\mathrm{hBN}$ platelets have an average particle size (lateral) of $c a .200 \mathrm{~nm}$, which is in agreement to an independent literature report utilising an equivalent fabrication methodology. ${ }^{24}$ EDX mapping analysis was performed to offer insight into the elemental composition of the area shown in ESI Fig. 3.† Analysis of the EDX map shows uniform distribution of $\mathrm{B}$ and $\mathrm{N}$ atoms with a ratio $(1: 1)$ of 20.12 at\% and 18.81 at\% B: $\mathrm{N}$ respectively and this composition correlates with expected values for surfactant exfoliated 2D-hBN. ${ }^{28}$

\section{Results and discussion}

\section{Surfactant exfoliated 2D-hBN utilised towards the electrochemical detection of DA, AA and UA}

The electron transfer reactions between surfactant exfoliated 2D-hBN and DA, AA and UA were first studied separately using unmodified SPEs in order to benchmark the electrochemical system. Fig. 1B-D depicts typical cyclic voltammograms obtained towards the electrochemical oxidation of $1 \mathrm{mM}$ DA, AA and UA respectively (separately in $\mathrm{pH} 7.4$ phosphate buffer solution (PBS)). When utilising unmodified/bare SPEs, the anodic oxidation potentials are observed at $+0.36 \mathrm{~V},+0.38 \mathrm{~V}$ and $+0.49 \mathrm{~V}$ respectively for the electrochemical oxidation of DA, AA and UA. This agrees well with previous literature. ${ }^{29}$

The electrochemical oxidation of DA, AA and UA was next considered using SPEs modified with increasing masses of surfactant exfoliated 2D-hBN (a coverage study), ranging from 7.5 to $300 \mathrm{ng}$. Fig. 1B depicts typical cyclic voltammograms obtained towards DA electrochemical oxidation at $150 \mathrm{ng}$ surfactant exfoliated 2D-hBN modified SPEs, where the potential required for oxidation decreases upon immobilisation of $2 \mathrm{D}$ hBN when compared to the response at an unmodified SPE. Analysis of these voltammograms is presented in ESI Fig. 5A 


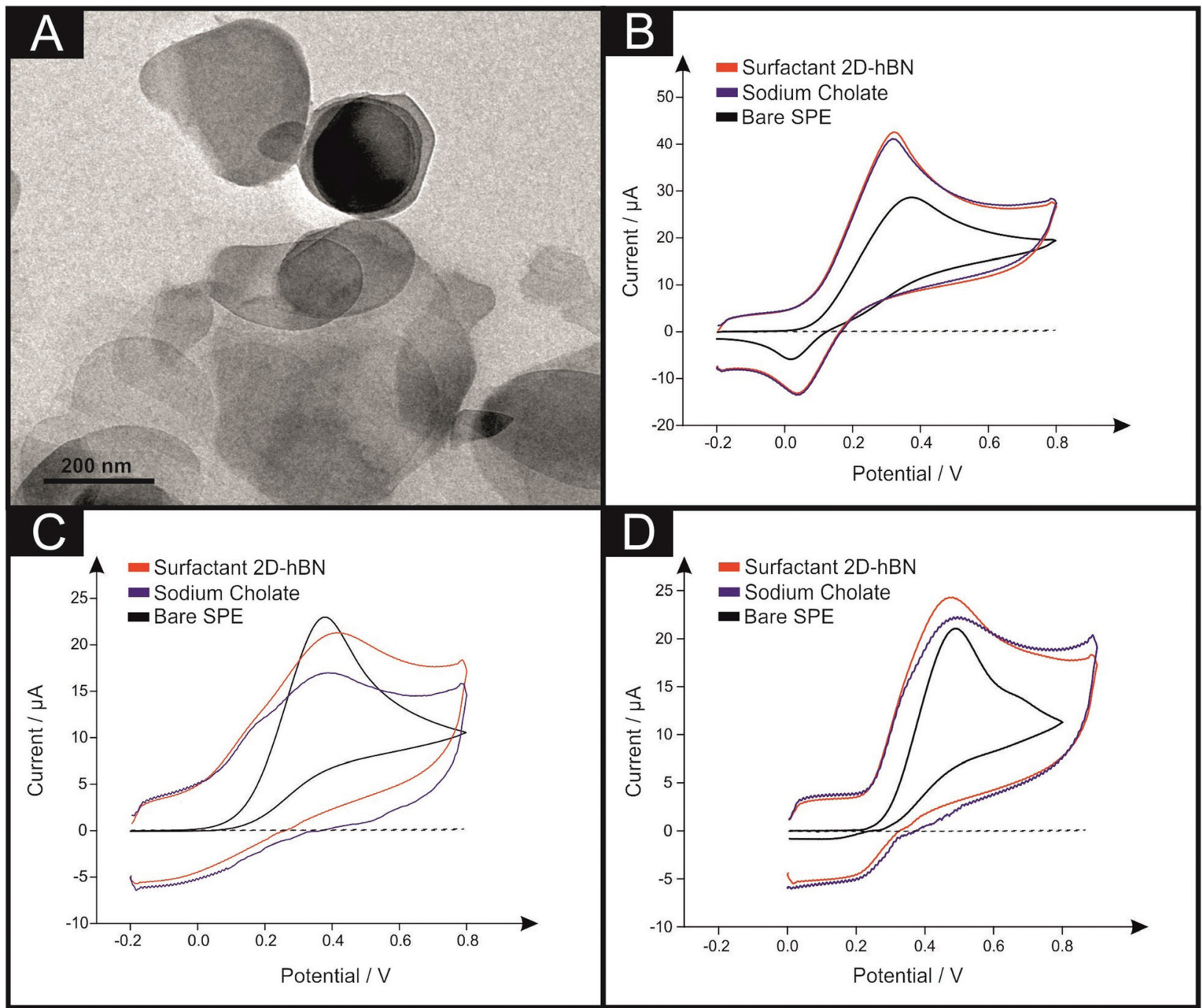

Fig. 1 Typical TEM image of surfactant exfoliated 2D-hBN after deposition onto a holey carbon film supported upon a Cu TEM grid (A). Also shown are typical cyclic voltammograms recorded in pH 7.4 PBS with (B) 1 mM DA, (C) 1 mM AA and (D) 1 mM UA when utilising unmodified SPEs (black), and $150 \mathrm{ng}$ surfactant exfoliated 2D-hBN (red) and $40 \mu \mathrm{g}$ sodium cholate (blue) modified SPEs. The dashed line represents the blank PBS respectively. Scan rate: $100 \mathrm{mV} \mathrm{s}^{-1}$ (vs. SCE). Note that full coverage study data of the modified electrodes is presented in ESI Fig. 5 and ESI Table $2 . \dagger$

and ESI Table $2, \uparrow$ where an overall decrease in DA oxidation potential of $c a .40 \mathrm{mV}$ (compared to an unmodified SPE) is exhibited. Furthermore, the observed peak currents evidently improve upon the immobilisation of increasing surfactant exfoliated 2D-hBN masses (ESI Fig. 5B and ESI Table 2†). Specifically, the current increases from 28.0 $\mu \mathrm{A}$ (unmodified SPE) to $35.8 \mu \mathrm{A}$ at a surfactant exfoliated $2 \mathrm{D}-\mathrm{hBN}$ modification mass of $150 \mathrm{ng}$. Note, the peak currents observed herein (measured at the baseline of each voltammogram respectively), are not as a result of increased capacitance upon the utilisation of surfactant exfoliated 2D-hBN modified SPEs, which is discussed later. Interestingly, we have previously shown that surfactant-free (pristine) 2D-hBN immobilised upon SPEs offers a greater, more beneficial response towards DA oxidation, reducing the potential by $c a .90 \mathrm{mV}^{20}$ Thus, to rationalise the observed difference in the DA anodic oxidation potentials and increased peak currents, control experiments were performed utilising sodium cholate modified SPEs (in the absence of $2 \mathrm{D}$-hBN) towards DA oxidation in order to understand and de-convolute the role said surfactant, which may potentially partake in the electrochemical process. Fig. 1B depicts a typical cyclic voltammogram of a $20 \mu \mathrm{g}$ sodium cholate modified SPE utilised towards DA oxidation. It is important to note that (throughout this study), the volume of surfactant utilised equates to the identical volume present within the specific and comparative surfactant exfoliated 2DhBN modification that was drop-cast onto the electrode surface, such that the true effect of the sodium cholate can be evaluated. The sodium cholate utilised for control experiments and exfoliation was also identical (see Experimental section). Returning to the observed electrochemical response, it is apparent within Fig. 1B that a similar voltammetric signature towards DA oxidation ( $c a .0 .32 \mathrm{~V}$ ) is observed at both the $150 \mathrm{ng}$ surfactant exfoliated $2 \mathrm{D}-\mathrm{hBN}$ and the equivalent 
sodium cholate (with no $2 D-h B N$ present) modified SPEs. Detailed analysis is presented in ESI Fig. 5 and ESI Table $2 \dagger$ (coverage study), where SPEs modified with sodium cholate (mass range: $2-80 \mu \mathrm{g}$ ) give rise to similar electrochemical oxidation potentials and peak currents towards DA detection as the equivalent surfactant exfoliated 2D-hBN modified SPEs. This is a significant observation, which suggests that the surfactant (sodium cholate) has a dominant role in the observed voltammetry recorded towards DA oxidation. Thus it is suggested the improved peak currents of DA in the anodic region and the increased capacitive response when utilising surfactant exfoliated 2D-hBN and its sodium cholate modified SPE equivalent (in comparison to unmodified SPEs) is likely due to the oxidation of cyclohexanol (hydroxyl groups) of the surfactant itself (Fig. 1B, ESI Fig. 5 and ESI Table $2 \dagger$ ). Interestingly, in this case (given the 'greater' beneficial response recorded at pristine $2 \mathrm{D}-\mathrm{hBN}$ with no surfactant present), the incorporation of the surfactant has an inhibiting effect on the electrochemistry of $2 \mathrm{D}-\mathrm{BN}$, as evidenced by the response shown upon the utilisation of pristine $2 \mathrm{D}-\mathrm{hBN}$ modified SPEs upon direct comparison with the use of surfactant exfoliated 2D-hBN modified SPEs. ${ }^{20}$

Equivalent studies to the aforementioned oxidation of DA were carried out towards the detection of AA and UA, utilising a range of equivalent surfactant exfoliated 2D-hBN (7.5-300 ng) and sodium cholate $(2-80 \mu \mathrm{g})$ modified SPEs. Inspection of Fig. 1C and D reveals that a $150 \mathrm{ng}$ surfactant exfoliated 2D-hBN modified SPE increases the electrochemical oxidation potential of AA by ca. $30 \mathrm{mV}$ and decreases the peak potential of UA by ca. $20 \mathrm{mV}$, whilst the peak currents decrease to $13.4 \mu \mathrm{A}$ and increase to $20.6 \mu \mathrm{A}$ respectively (when compared to the unmodified SPEs, vide supra). Detailed analysis is shown in ESI Fig. 5A and $5 \mathrm{~B}$ and ESI Table $2 \dagger$ where coverage studies reveal the inhibitory/detrimental effect of utilising surfactant exfoliated 2D-hBN modified SPEs towards the detection of AA and UA. We have previously reported that pristine $2 \mathrm{D}-\mathrm{hBN}$ modified SPEs exhibit a detrimental effect towards the detection of AA; inferred due to the oxidation mechanism of AA on carbon-based electrodes being invoked at specific functionalities and oxygenated surface sites (which 2D-hBN likely blocks). ${ }^{20,30}$ However, the case of UA detection was somewhat different, with pristine $2 \mathrm{D}$ hBN modified SPEs exhibiting a beneficial response in terms of the potential required for its anodic oxidation (with a decrease of up to $c a .50 \mathrm{mV}$ shown). ${ }^{20}$ To de-convolute the inhibitory response of surfactant exfoliated 2D-hBN modified SPEs, control studies in the form of equivalent sodium cholate modified SPEs were carried out. It is clear, as previously observed with DA oxidation, that similar anodic peak potentials and currents are exhibited at the surfactant modified SPEs when compared directly to the surfactant exfoliated 2D-hBN equivalents. This work suggests that the sodium cholate is interfering and potentially dominating the electrochemical response in the former case (when present with the 2D-hBN), thus resulting in 2D-hBN's apparent not so beneficial response.

Next, the simultaneous detection of DA and AA was investigated via cyclic voltammetry by adding equal aliquots of DA and $\mathrm{AA}$ at the equal concentration of $0.5 \mathrm{mM}$ into a $\mathrm{pH} 7.4$ PBS. First, unmodified SPEs were utilised and the observed response is depicted in Fig. 2A. It is clear that in this case the simultaneous detection of DA and AA is not viable, with a single oxidation peak observed at $c a$. $+0.32 \mathrm{~V}$ (peak current: 15.7 $\mu \mathrm{A}$ ). Modification of the SPE with $150 \mathrm{ng}$ surfactant exfoliated $2 \mathrm{D}-\mathrm{hBN}$ results in an increased peak current of 24.5 $\mu \mathrm{A}$ and a reduced peak potential for the DA and AA oxidations $(\mathrm{ca} .+0.25 \mathrm{~V})$, however the simultaneous detection is again not viable. A similar response towards the simultaneous detection of DA and AA was reported previously when utilising pristine $2 \mathrm{D}-\mathrm{hBN}$ (no surfactant) modified SPEs, suggesting that 2D-hBN itself blocks the oxidation mechanism of AA when it is immobilised upon carbon-based electrodes. ${ }^{20,30}$ To confirm this insight, a control experiment was performed utilising a $40 \mu \mathrm{g}$ sodium cholate modified SPE (equivalent surfactant content as per the surfactant exfoliated 2D-hBN). As evident within Fig. 2A, a similar response is observed towards the simultaneous detection of DA and AA at both the surfactant exfoliated 2D-hBN and the surfactant (control: no 2D-hBN present) modified SPE. Specifically, a single peak is depicted at $c a .0 .24 \mathrm{~V}$ and a maximum current of $22.5 \mu \mathrm{A}(40 \mu \mathrm{g}$ sodium cholate modified SPE). Evidently, the surfactant present interferes and dominates the electrochemical response observed when utilising 2D-hBN.

We now investigate this further and turn our attention towards the simultaneous detection of DA and UA. Cyclic voltammetry was performed after adding equal aliquots of DA and UA (at a concentration of $0.5 \mathrm{mM}$ ) into a $\mathrm{pH} 7.4$ PBS. Fig. 2B shows that the simultaneous detection of DA and UA is viable when utilising an unmodified SPE, with the respective oxidation peaks observed at ca. $+0.28(7.48 \mu \mathrm{A})$ and $+0.51 \mathrm{~V}$ $(9.36 \mu \mathrm{A})$. Modification of the SPEs with $150 \mathrm{ng}$ surfactant exfoliated $2 \mathrm{D}-\mathrm{hBN}$ results in a lower (less electro-positive, i.e. beneficial response) oxidation peak potential of $+0.22 \mathrm{~V}$, with an increased peak current of $16.7 \mu \mathrm{A}$ for DA (when compared to that of an unmodified SPE). However, a detrimental response is observed in the case of UA, with a similar peak potential exhibited $(+0.50 \mathrm{~V})$ and a lower peak current $(7.28 \mu \mathrm{A})$, suggesting a decrease in sensitivity. This observation differs considerably to our previous work that utilised pristine $2 \mathrm{D}$ hBN towards the simultaneous detection of DA and UA, where the peak separation/resolution of both anodic oxidation peaks was enhanced with the inclusion of $2 \mathrm{D}-\mathrm{hBN} .^{20}$ To de-convolute the response, an equivalent sodium cholate control study was performed with a $40 \mu \mathrm{g}$ modified SPE. Fig. 2B depicts the response obtained towards DA and UA oxidation, demonstrating a similar response to exhibited at the $150 \mathrm{ng}$ surfactant exfoliated 2D-hBN modified SPE, with respective oxidation peaks observed at $c a .+0.22 \mathrm{~V}(14.9 \mu \mathrm{A})$ and $+0.52 \mathrm{~V}(7.11 \mu \mathrm{A})$. Evidently, the inclusion of the surfactant does not offer a favourable interaction between the analyte of interest and the electrode surface, resulting in the significantly inhibited electrochemical oxidation process of UA. ${ }^{25}$ It is clear that the surfactant utilised in the exfoliation of $2 \mathrm{D}-\mathrm{hBN}$ hinders the observed electrochemical response towards the simultaneous detection of DA and UA, where, given the previous reports at 


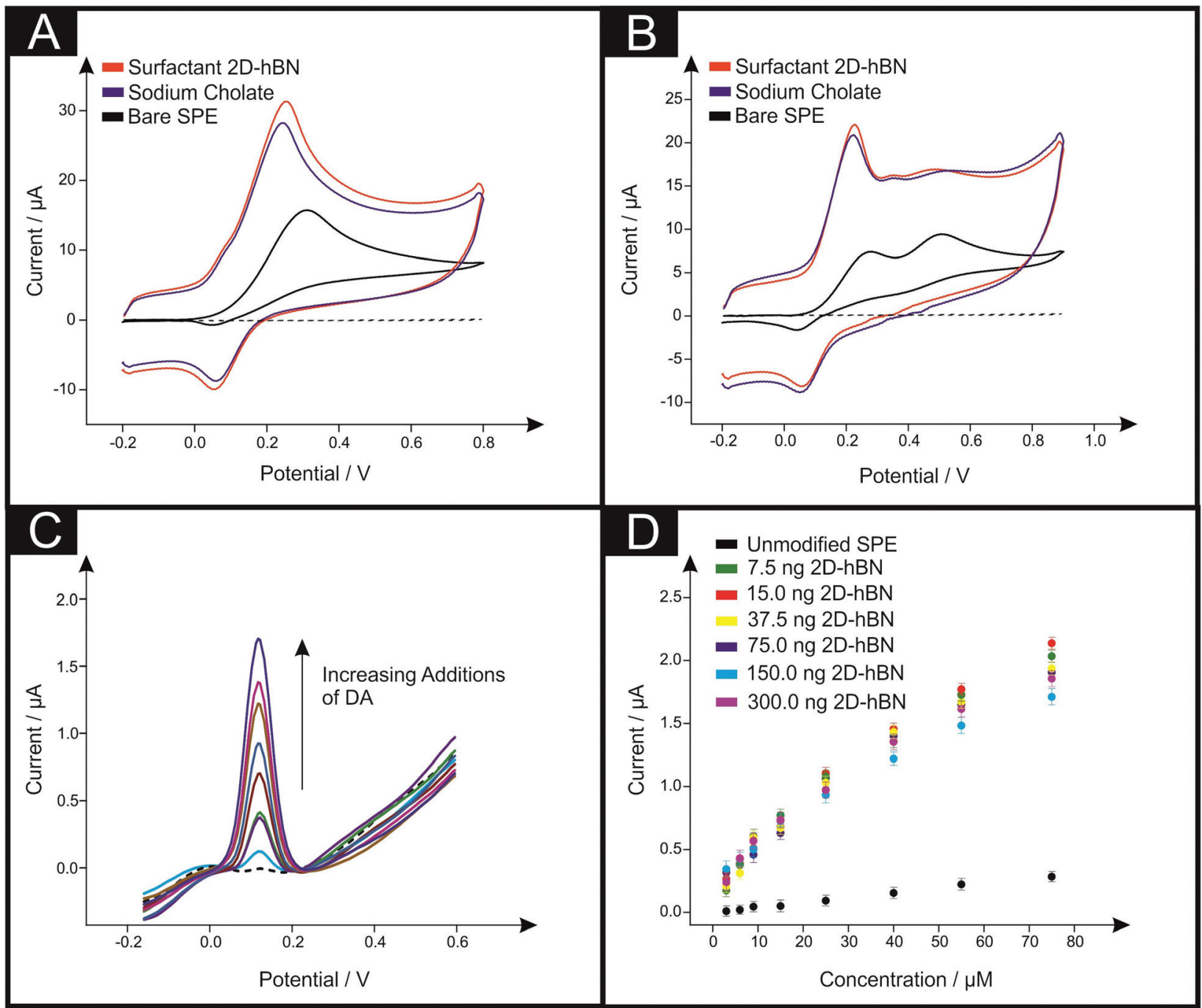

Fig. 2 (A) Typical cyclic voltammograms recorded in $0.5 \mathrm{mM} \mathrm{DA}$ and $0.5 \mathrm{mM}$ AA (PBS pH 7.4) using an unmodified (black), $150 \mathrm{ng}$ surfactant exfoliated 2D-hBN (red) and $40 \mu \mathrm{g}$ sodium cholate (blue) modified SPEs. (B) Voltammograms recorded in $0.5 \mathrm{mM}$ DA and $0.5 \mathrm{mM}$ UA (PBS pH 7.4 ) at unmodified (black), $150 \mathrm{ng}$ surfactant exfoliated 2D-hBN (red) and $40 \mu \mathrm{g}$ sodium cholate (blue) modified SPEs. A and B were recorded utilising a scan rate of $100 \mathrm{mV} \mathrm{s}^{-1}$ (vs. SCE). (C) Typical DPVs obtained by adding aliquots of DA at concentrations in the range of 3-75 $\mu \mathrm{M}$ into a $0.1 \mathrm{mM}$ AA solution (pH 7.4 PBS) utilising a 150 ng surfactant exfoliated 2D-hBN modified SPE; the dashed line represents $0.1 \mathrm{mM}$ AA with no DA present. (D) Analytical curves corresponding to the anodic peak current for the oxidation of DA over the concentration range studied, obtained utilising unmodified and surfactant exfoliated 2D-hBN (mass range: 7.5-300 ng) modified SPEs. DPV conditions: $E$-pulse, $20 \mathrm{mV} ; t$-pulse, $200 \mathrm{~ms}$; equivalent scan rate, $10 \mathrm{mV} \mathrm{s}^{-1}$; (vs. SCE). Each data (D) is the average deviation $(N=3)$.

pristine 2D-hBN modified SPEs, the electrocatalytic effect of $2 \mathrm{D}$-hBN is masked. Evidently, the response observed herein (and in other reports within the literature that utilise similar surfactants) should not be assumed to be due solely to 2DhBN itself, but rather due to the effect of the surfactant present (sodium cholate) or other contributing factors incorporated as part of the fabrication process of 2D-hBN (and other novel 2D materials), that, as we have shown, can contribute to and dominate the electrochemical response.

Note that there is significant signal overlap of DA and AA and therefore the simultaneous detection of all three analytes (DA, AA and UA) is not viable with our approach. Resultantly, we do not consider the case of all three compounds but focus herein on the simultaneous detection of DA in the presence of AA and UA separately.

Differential pulse voltammetry (DPV) was next utilised to further scrutinise the simultaneous detection of DA and AA, with aliquots of DA added (representing, 3-75 $\mu \mathrm{M}$ ) into a $0.1 \mathrm{mM}$ AA solution. ESI Fig. $6 \mathrm{~A} \dagger$ shows the observed voltammetry at unmodified SPEs, where upon additions of DA an increment in the current relating to the oxidation of DA is observed (with a small displacement of peak potentials to more electronegative regions). Again, the simultaneous detection of DA and AA is not observed when utilising an unmodified/bare SPE.

Next, a range of surfactant exfoliated 2D-hBN masses (7.5-300 ng) were immobilised upon SPEs. Fig. 2C depicts 
typical DPVs obtained towards the attempted simultaneous detection of DA and AA utilising $150 \mathrm{ng}$ surfactant exfoliated 2D-hBN modified SPEs. In this case, additions of DA resulted in a significant increase in the peak current obtained for DA oxidation when compared to an unmodified SPE. However, again, a single oxidation peak is evident, indicating the unviability of simultaneous DA and AA detection. Of note however is that the oxidation potential does not differ upon DA additions in this latter case, contrasting the behaviour observed at unmodified SPEs. This suggests that the electrode (following modification of the surface with surfactant exfoliated 2D-hBN) is less susceptible to oxidised species adsorbing onto its surface. Further analysis of this is depicted in Fig. 2D, with a range of surfactant exfoliated $2 \mathrm{D}-\mathrm{hBN}$ modified SPEs (7.5-300 ng) illustrating the differences exhibited in the linear calibration curves at these modified SPEs. What is clear through inspection of Fig. 2D, is that immobilisation of surfactant exfoliated 2D-hBN increases the analytical sensitivity towards the detection of DA (when compared to the unmodified $\mathrm{SPE}$ ). Interestingly however, when further mass-additions are employed, the beneficial response reduces after a mass of 150 $\mathrm{ng}$ is utilised. Previous studies utilising pristine $2 \mathrm{D}-\mathrm{hBN}$ modified SPEs towards the simultaneous detection of DA and AA gave rise to a similar response, suggesting that $2 \mathrm{D}-\mathrm{hBN}$ is not a viable candidate for the simultaneous detection of DA and AA, although it does offer improved electroanalytical signals towards DA. ${ }^{20}$ To rationalise this further, a sodium cholate control study was carried out (see ESI Fig. $7 \dagger$ ) which demonstrated a similar response to that obtained at the surfactant exfoliated 2D-hBN modified SPEs. Again, it is suggested that sodium cholate (the surfactant utilised) is likely dominating the observed electrochemical response. We offer insight into the possible mechanism of this response below (vide infra). Likely due to either favourable surface charges or the oxidation of cyclohexanol on the surfactant structure, ${ }^{25,31-33}$ where in the case of the electrochemical oxidation of DA there could be a favourable interaction between the electrochemical product formed and the surfactant itself, which may be the opposite case for AA where no such beneficial effect is exhibited.

Finally, attention was directed towards the simultaneous detection of DA and UA when utilising DPV, with aliquots of DA added (representing, 3-75 $\mu \mathrm{M}$ ) into a $0.1 \mathrm{mM}$ UA solution. ESI Fig. $6 \mathrm{~B} \uparrow$ shows the observed voltammetry at unmodified SPEs, where upon additions of DA an increase in the current relating to the oxidation of DA is observed, with a signal for UA oxidation also apparent. Accordingly, the simultaneous of detection of DA and UA is viable at unmodified SPEs. We therefore consider utilising $150 \mathrm{ng}$ surfactant exfoliated 2DhBN modified SPEs. ESI Fig. $8 \mathrm{~A} \uparrow$ depicts the voltammograms obtained with additions of DA resulting in a significant increase in the peak current obtained corresponding to the oxidation of DA (when contrasted to an unmodified SPE). However, interestingly, it is apparent that in the case of utilising surfactant exfoliated 2D-hBN, increasing additions of DA results in a reduced peak current observed for UA. Further analysis of this is presented in ESI Fig. $8 \mathrm{~B}$ and $\mathrm{C}, \dagger$ where a range of surfactant exfoliated $2 \mathrm{D}-\mathrm{hBN}$ masses $(7.5-300 \mathrm{ng}$ ) were immobilised upon SPEs and explored towards the simultaneous detection of DA and UA. It is clear the lowest mass immobilisation of surfactant 2D-hBN (7.5 ng) offers the greatest beneficial response corresponding to the peak height of the DA signal. With further increases in the mass of surfactant exfoliated 2D-hBN immobilised upon an SPE decreasing the peak currents observed, whilst simultaneously consistently reducing the peak currents of UA. This suggests improved sensitivity towards the electrochemical oxidation of DA in comparison to an unmodified SPE, whereas the opposite is evident towards UA detection. Further analysis of this behaviour was carried out in the form of sodium cholate control coverage studies $(2-80 \mu \mathrm{g})$, which are shown in ESI Fig. 9A-C, $\dagger$ demonstrating a similar trend to that of the surfactant exfoliated 2DhBN modified SPEs. We have previously shown that the use of pristine 2D-hBN improves the peak separation/resolution towards the simultaneous detection of DA and UA. ${ }^{20}$ With this in mind, it is clear upon the utilisation of surfactant exfoliated 2D-hBN modified SPEs, that a detrimental effect towards the said detection is exhibited, particularly in the case of UA. Therefore, it is inferred that the exfoliation process of $2 \mathrm{D}-\mathrm{hBN}$ (i.e. the use of surfactants) is the origin of the distinct change in behaviour. Clearly, it is of vital importance to consider the surfactant utilised in the exfoliation of $2 \mathrm{D}-\mathrm{hBN}$ in order to deconvolute the true electrochemical response of this material.

Next, the limit of detection (LOD, based on three-sigma) for surfactant exfoliated 2D-hBN was calculated and the $300 \mathrm{ng}$ 2D-hBN modified SPE exhibited a LOD for DA (in the presence of UA) of $1.57 \mu \mathrm{M}$, which is higher than that of the previously studied pristine $2 \mathrm{D}-\mathrm{hBN}(0.65 \mu \mathrm{M})$, suggesting the surfactant utilised in the exfoliation of $2 \mathrm{D}-\mathrm{hBN}$ can hinder its analytical utility. A full comparison with other 2D materials is shown in Table 1. On the other hand; the unmodified SPE possesses a LOD of $2.89 \mu \mathrm{M}$.

Interestingly, sodium cholate has been utilised previously towards the surfactant based exfoliation of graphene, where subsequent studies demonstrated that, when present, the surfactant contributes to (and in some cases dominates) the observed electrochemistry. ${ }^{25,32,33}$ In the aforementioned studies, the surfactant (sodium cholate) was shown to be electroactive, in that it gave rise to an electrochemical oxidation peak at $c a .+0.30 \mathrm{~V}$ when modified onto SPEs and cyclic voltammetry was performed in blank buffers. ${ }^{25}$ Based upon the chemical structure, it is surmised that the electrochemical response is due to the oxidation of cyclohexanol (hydroxyl groups), via an equal proton/electron transfer process (which is likely to be 2). Thus, in our case, where we observe an improved peak current in the anodic region, it is likely that oxidation of the surfactant itself contributes to this increment. However, the effects of surfactants are complicated and we observe a variety of different responses when utilising distinct analytes (i.e. DA, UA and $\mathrm{AA}$ ). In other studies, the charges present on various surfactants (including sodium cholate) have been shown to interfere with the observed electrochemical response ${ }^{31,32}$ for example, inhibiting metal sensing 
and alternatively improving aerometric biosensors. It is likely that the influence of surface charges (and/or potentially a change in hydrophilicity) on the electrode surface is contributing to the response observed in this work, which are (in this case) detrimental and thus inferred to result in unfavourable interactions between the electrochemical analyte/product with that of the surfactant/electrode surface. The effect of surfactants on electrochemical processes are not completely understood and more work is needed to elucidate the exact mechanism. ${ }^{25,34,35}$ What is clear however, is that the surfactant utilised in the exfoliation of $2 \mathrm{D}-\mathrm{hBN}$ contributes to (and in some cases dominates) the observed electrochemical response. Thus, as reported herein, when utilising surfactant exfoliated 2D-hBN, the electrochemical response should not be assumed to be due solely to $2 \mathrm{D}$-hBN itself, but rather due to the effect of the surfactant (sodium cholate) incorporated as part of the fabrication process, that can contribute to and dominate the electrochemical response. This applies also to other newly emerging novel 2D materials and to other contributing factors incorporated as part of the fabrication process. Researchers in this field should be cautious (particularly before reporting beneficial electrocatalysis) and ensure that diligent control experiments (such as those reported herein) are performed.

\section{Conclusions}

This paper has explored, for the first time, the use of surfactant exfoliated 2D-hBN nanosheets (immobilised upon a supporting SPE surface) as a potential electrochemical sensing platform towards the simultaneous detection of DA, AA and UA. The effects of 'mass coverage' was explored on the 2DhBN's electrochemical behaviour and was shown to be an essential consideration when investigating novel nanomaterials that should be implemented as routine protocols to fully understand and ascertain the true properties. Furthermore, through the implementation of diligent control experiments (in the form of equivalently modified surfactant electrodes, with no $2 \mathrm{D}$-hBN present), we are able to report that the fabrication route utilised to prepare said $2 \mathrm{D}-\mathrm{hBN}$ is a vital experimental consideration that is often overlooked within the literature. We have shown that the surfactant utilised in the exfoliation of $2 \mathrm{D}-\mathrm{hBN}$ contributes to (and dominates) the observed electrochemical response. When utilising surfactant exfoliated 2D-hBN, we urge researchers within the field to consider a contribution from the inherent surfactant. We infer that the electrochemical response should not be assumed to be due solely to $2 \mathrm{D}$-hBN itself, but rather due to the effect of surfactant (in this case, sodium cholate) incorporated as part of the fabrication process. Given the wide variety of ionic and non-ionic surfactants that are utilised in the manufacture of novel 2D materials, these findings should be applied to other newly emerging 2D materials (and the various surfactants utilised) and should serve as an important warning when considering other contributing factors incorporated as part of the various fabrication processes. ${ }^{25}$ It is likely that different surfac- tant and 2D material combinations will give rise to distinct relationships and as such, the electrochemical performance/ properties will be affected in different ways, either beneficially or detrimentally. ${ }^{25}$ Critically, when investigating the electrochemical properties/performance of such materials, researchers should exercise caution (particularly before reporting beneficial electrocatalysis) and ensure that diligent control experiments (such as those reported herein), are performed in order to effectively de-convolute the observed response and correctly attribute the individual contributions. The significance here is not in the material itself, but the fundamental knowledge that the surfactant (preparation/synthesis method) and surface coverage utilised changes the electrochemical properties of the material under investigation.

\section{Acknowledgements}

The authors acknowledge funding from the Engineering and Physical Sciences Research Council (Reference: EP/N001877/1) and a British Council Institutional Grant Link (No. 172726574). D. A. C. Brownson acknowledges funding from the Ramsay Memorial Fellowships Trust.

\section{References}

1 P. Wang, Y. Li, X. Huang and L. Wang, Talanta, 2007, 73, 431-437.

2 B. A. J. Larkin, M. El-Sayed, D. A. C. Brownson and C. E. Banks, Anal. Methods, 2012, 4, 721-729.

3 H. Sun, J. Chao, X. Zuo, S. Su, X. Liu, L. Yuwen, C. Fan and L. Wang, RSC Adv., 2014, 4, 27625-27629.

4 S. R. Ali, Y. Ma, R. R. Parajuli, Y. Balogun, W. Y. C. Lai and H. He, Anal. Chem., 2007, 79, 2583-2587.

5 A. Pandikumar, G. T. Soon How, T. P. See, F. S. Omar, S. Jayabal, K. Z. Kamali, N. Yusoff, A. Jamil, R. Ramaraj, S. A. John, H. N. Lim and N. M. Huang, RSC Adv., 2014, 4, 63296-63323.

6 L. C. S. Figueiredo-Filho, D. A. C. Brownson, O. FatibelloFilho and C. E. Banks, Analyst, 2013, 138, 4436-4442.

7 H.-X. Zhao, H. Mu, Y.-H. Bai, H. Yu and Y.-M. Hu, J. Pharm. Anal., 2011, 1, 208-212.

8 L. C. S. Figueiredo-Filho, D. A. C. Brownson, O. FatibelloFilho and C. E. Banks, Electroanalysis, 2014, 26, 93-102.

9 K. Jackowska and P. Krysinski, Anal. Bioanal. Chem., 2012, 405, 3753-3771.

10 D. A. C. Brownson, L. C. S. Figueiredo-Filho, B. L. Riehl, B. D. Riehl, M. Gomez-Mingot, J. Iniesta, O. Fatibello-Filho and C. E. Banks, J. Mater. Chem. A, 2016, 4, 2617-2629.

11 R. A. Medeiros, A. Benchick, R. C. Rocha-Filho, O. Fatibello-Filho, B. Saidani, C. Debiemme-Chouvy and C. Deslouis, Electrochem. Commun., 2012, 24, 61-64.

12 Y. Wang and Y. Xiao, Microchim. Acta, 2012, 178, 123-130. 
13 R. A. Medeiros, R. Matos, A. Benchikh, B. Saidani, C. Debiemme-Chouvy, C. Deslouis, R. C. Rocha-Filho and O. Fatibello-Filho, Anal. Chim. Acta, 2013, 797, 30-39.

14 C. Deng, J. Chen, M. Wang, C. Xiao, Z. Nie and S. Yao, Biosens. Bioelectron., 2009, 24, 2091-2094.

15 H. Filik, A. A. Avan and S. Aydar, Arab. J. Chem., 2016, 9, 471-480.

16 M. D. Hawley, S. V. Tatawawadi, S. Piekarski and R. N. Adams, J. Am. Chem. Soc., 1967, 89, 447-450.

17 D. Han, T. Han, C. Shan, A. Ivaska and L. Niu, Electroanalysis, 2010, 22, 2001-2008.

18 Z.-H. Sheng, X.-Q. Zheng, J.-Y. Xu, W.-J. Bao, F.-B. Wang and X.-H. Xia, Biosens. Bioelectron., 2012, 34, 125-131.

19 X. Tian, C. Cheng, H. Yuan, J. Du, D. Xiao, S. Xie and M. M. F. Choi, Talanta, 2012, 93, 79-85.

20 A. F. Khan, D. A. C. Brownson, E. P. Randviir, G. C. Smith and C. E. Banks, Anal. Chem., 2016, 88, 9729-9737.

21 Y.-R. Kim, S. Bong, Y.-J. Kang, Y. Yang, R. K. Mahajan, J. S. Kim and H. Kim, Biosens. Bioelectron., 2010, 25, 23662369.

22 E. Blanco, C. W. Foster, L. R. Cumba, D. R. do Carmo and C. E. Banks, Analyst, 2016, 141, 2783-2790.

23 L. R. Cumba, C. W. Foster, D. A. C. Brownson, J. P. Smith, J. Iniesta, B. Thakur, D. R. do Carmo and C. E. Banks, Analyst, 2016, 141, 2791-2799.

24 R. Kurapati, C. Backes, C. Ménard-Moyon, J. N. Coleman and A. Bianco, Angew. Chem., Int. Ed., 2016, 55, 55065511.

25 D. A. C. Brownson, J. P. Metters, D. K. Kampouris and C. E. Banks, Electroanalysis, 2011, 23, 894-899.

26 J. Tauc, R. Grigorovici and A. Vancu, Phys. Status Solidi B, 1966, 15, 627-637.
27 A. F. Khan, M. P. Down, G. C. Smith, C. W. Foster and C. E. Banks, J. Mater. Chem. A, 2017, 5, 4103-4113.

28 A. F. Khan, E. P. Randviir, D. A. C. Brownson, X. Ji, G. C. Smith and C. E. Banks, Electroanalysis, 2017, 29, 622634.

29 E. P. Randviir, D. A. C. Brownson, J. P. Metters, R. O. Kadara and C. E. Banks, Phys. Chem. Chem. Phys., 2014, 16, 4598-4611.

30 I. F. Hu and T. Kuwana, Anal. Chem., 1986, 58, 3235-3239.

31 D. A. C. Brownson and C. E. Banks, Electrochem. Commun., 2011, 13, 111-113.

32 D. A. C. Brownson and C. E. Banks, Analyst, 2011, 136, 2084-2089.

33 D. A. C. Brownson and C. E. Banks, Chem. Commun., 2012, 48, 1425-1427.

34 M. Pumera, R. Scipioni, H. Iwai, T. Ohno, Y. Miyahara and M. Boero, Chem. - Eur. J., 2009, 15, 10851-10856.

35 J. F. Rusling, Colloids Surf., A, 1997, 123, 81-88.

36 M. Mallesha, R. Manjunatha, C. Nethravathi, G. S. Suresh, M. Rajamathi, J. S. Melo and T. V. Venkatesha, Bioelectrochemistry, 2011, 81, 104-108.

37 J. Chao, X. Han, H. Sun, S. Su, L. Weng and L. Wang, Sci. China Chem., 2016, 59, 332-337.

38 S. Su, H. Sun, F. Xu, L. Yuwen and L. Wang, Electroanalysis, 2013, 25, 2523-2529.

39 Y.-R. Kim, S. Bong, Y.-J. Kang, Y. Yang, R. K. Mahajan, J. S. Kim and H. Kim, Biosens. Bioelectron., 2010, 25, 23662369.

40 V. Mani, M. Govindasamy, S.-M. Chen, R. Karthik and S.-T. Huang, Microchim. Acta, 2016, 183, 2267-2275.

41 K. Pramoda, K. Moses, U. Maitra and C. N. R. Rao, Electroanalysis, 2015, 27, 1892-1898. 